\title{
Modern evidence and future prospects of external body radiation therapy for lung oligometastases of breast cancer
}

\author{
Yuki Wada, Manabu Hashimoto \\ Department of Radiology, Akita University Graduate School of Medicine, 1-1-1 Hondo, Akita, Akita 010-8543, Japan \\ Contributions: (I) Conception and design: All authors; (II) Administrative support: None; (III) Provision of study materials or patients: None; (IV) \\ Collection and assembly of data: None; (V) Data analysis and interpretation: All authors; (VI) Manuscript writing: All authors; (VII) Final approval of \\ manuscript: All authors \\ Correspondence to: Yuki Wada. Department of Radiology, Akita University Graduate School of Medicine, 1-1-1 Hondo, Akita, Akita 010-8543, Japan. \\ Email: ywada@med.akita-u.ac.jp.
}

\begin{abstract}
After Hellman and Weichselbaum defined "Oligometastasis" in 1995, several local therapies for lung oligometastases including surgical resection and external body radiation therapy were reported that improved local control (LC) and progression-free survival, overall survival, and quality of life. This suggests that oligometastases is a potentially curable state. Modern advances in radiation therapy such as stereotactic body radiation therapy (SBRT) in which high dose coverage of target lesion without exposure of normal organ is possible, and are widely used to treat solitary or a limited number of primary lung cancer and metastases. Several reports showed that SBRT was a useful treatment method for lung oligometastases, and the LC rate of SBRT was $80-90 \%$ in 2 years and less invasive than surgical resection. SBRT is a safe and effective especially for small and peripheral lung metastases. However, if the metastatic lesion is big or centrally located, careful treatment is necessary to prevent radiation pneumonitis. After SBRT, it is sometimes difficult to differentiate local recurrence and pulmonary injury, especially in the early phase. However, it is important to detect local recurrence especially in patients who require further local therapy such as surgical resection and re-irradiation or systemic therapy. The diagnosis can be improved by determining the natural course after SBRT and local recurrence with computed tomography imaging and ${ }^{18} \mathrm{~F}$-fluorodeoxyglucose positron emission tomography, respectively. Moreover, radiation therapy may have both local and systemic effects that are related to the enhancement of immune-response after radiation. Currently, several trials evaluating the benefits of SBRT for oligometastatic breast cancer are underway. However, the adaption of SBRT for lung metastases including other treatment strategies should be carefully discussed by the radiation oncologist and a multi-disciplinary team comprising a breast surgeon, medical oncologist, diagnostic radiologist, and radiation oncologist, among others.
\end{abstract}

Keywords: Stereotactic body radiation therapy (SBRT); lung oligometastasis; stereotactic ablative radiation; external body radiation therapy

Submitted Jan 01, 2020. Accepted for publication Feb 12, 2020.

doi: $10.21037 /$ tcr.2020.02.55

View this article at: http://dx.doi.org/10.21037/tcr.2020.02.55

\section{Introduction}

The standard treatment strategy for metastatic breast cancer is systemic chemotherapy or hormone therapy that treats both gross lesions and microscopic metastatic lesions which cannot be detected in diagnostic images. However, these strategies cannot eradicate gross metastases, and hence, these are mainly focused on preventing progression and new metastasis and maintain the quality of life (QOL) but do not aim to cure. In 1995, Hellman and Weichselbaum were the first to define the innovative concept of "oligometastases" in which a single or a limited number of metastatic lesions exist that are not systemically widespread (1). Subsequently, 
Table 1 Differences between three-dimensional conformal radiation therapy (3D-CRT) and stereotactic body radiation therapy (SBRT)

\begin{tabular}{|c|c|c|}
\hline Methods of radiation & 3D-CRT & SBRT \\
\hline $\begin{array}{l}\text { Total fractions } \\
\text { (treatment duration) }\end{array}$ & $28-33$ fractions in $6-7$ weeks & Several protocols are used worldwide; approximately, within 1-2 weeks (7-11) \\
\hline Total dose & 60-66 Gy & $\begin{array}{l}\text { Several protocols are used worldwide; in Japan, } 48 \text { Gy in } 4 \text { fractions is most } \\
\text { frequently used; followed by } 50 \text { Gy in } 5 \text { fractions, and } 60 \text { Gy in } 8 \text { fractions ( } 7 \text {;) } \\
\text { usually, for central lesions, total dose and dose-per-fraction are modified for } \\
\text { example } 50 \text { Gy in } 5 \text { fractions and } 56 \text { Gy in } 7 \text { fractions, however, several } \\
\text { prospective trials are undergoing regarding the treatment of central lesions }\end{array}$ \\
\hline $\begin{array}{l}\text { Biological effective } \\
\text { dose } \alpha / \beta=10\left(B D_{10}\right)\end{array}$ & 72 Gy for 60 Gy/30 fractions & $\begin{array}{l}105.6 \text { Gy for } 48 \text { Gy/4 fractions, } 100 \text { Gy for } 50 \text { Gy/5 fractions; BED } 10>100 \text { Gy is } \\
\text { related with good prognosis }(12,13)\end{array}$ \\
\hline Dose contribution & $\begin{array}{l}\text { Band or box-shaped irradiation } \\
\text { area including target lesion; hence, } \\
\text { normal organs around the target are } \\
\text { widely included }\end{array}$ & $\begin{array}{l}\text { Almost target fitted irradiation area with minimal margin for the target; hence, } \\
\text { decrease dose of normal organ near the target is possible }\end{array}$ \\
\hline
\end{tabular}

oligometastases are widely known because of the modern advancement in both diagnostic methods and less-invasive treatment techniques. Local treatment for oligometastases such as surgical resection, radiofrequency ablation, and external beam radiation therapy (EBRT) are beneficial as they may be able to achieve complete response or "cure" patients such that further systemic therapy can be avoided. Several reports (2-4) showed that local treatment of oligometastases improves not only local control (LC) of metastatic lesions, progression-free survival (PFS), and overall survival (OS); hence, oligometastases are a potentially curable state and should be treated by a multidisciplinary team including breast surgeon, medical oncologist, diagnostic radiologist, and radiation oncologist, among others (5). EBRT as a local therapy for lung oligometastases was reported to have a good LC rate and was less invasive than surgical resection. Furthermore, in the case of stereotactic body radiation therapy (SBRT) for non-small cell lung cancer (NSCLC), there were only a few clinically significant changes in the health-related QOL before and after SBRT (6).

In this review article, we discuss the modern evidence and future prospects of EBRT, especially SBRT [or stereotactic ablative radiotherapy (SABR)]. Since the target audience is non-radiation oncologists, we omitted details regarding radiation planning and focused on clinically important aspects such as clinical outcomes, toxicities, follow-up after EBRT, and future prospects of SBRT. We hope that this article will be helpful for oncologists who are considering SBRT or following up patients after SBRT.

\section{Radiation technique and regimens for lung metastases: differences between three- dimensional conformal radiation therapy (3D-CRT) and SBRT}

Two kinds of EBRT methods are available for local therapy of lung oligometastases: 3D-CRT and SBRT/SABR. The different characteristics of these therapies are listed in Table 1, and Figure 1 shows the difference in dose contribution between these two methods. The most commonly used method to treat any kind of cancers is 3D-CRT. In this, the megavoltage $\mathrm{X}$-rays are fitted to the tumor shape by multi-leaf collimators which are located at the gantry of linear accelerator and are irradiated from 2-4 directions. However, in 3D-CRT, the irradiated area is shaped as a band or box, and therefore, normal organs around the target lesion are unnecessarily included in the irradiated area, as shown in Figure 1A. To decrease the side effects caused by the unnecessary irradiation of normal organs, the dose per fraction is limited to 1.8-2.0 Gy and irradiation accumulated up to approximately $60 \mathrm{~Gy}$ in 30 fractions for 6 weeks.

Modern advantages of radiation techniques such as image-guided radiation therapy and breath-gated radiation enabled the radiation oncologist to minimize the planning target volume (PTV) and irradiate with high accuracy. These techniques minimize the area of unnecessarily irradiated normal organs. In the case of SBRT/SABR, the radiation oncologists can plan a high dose coverage of target lesions and reduce radiation exposure of the area 

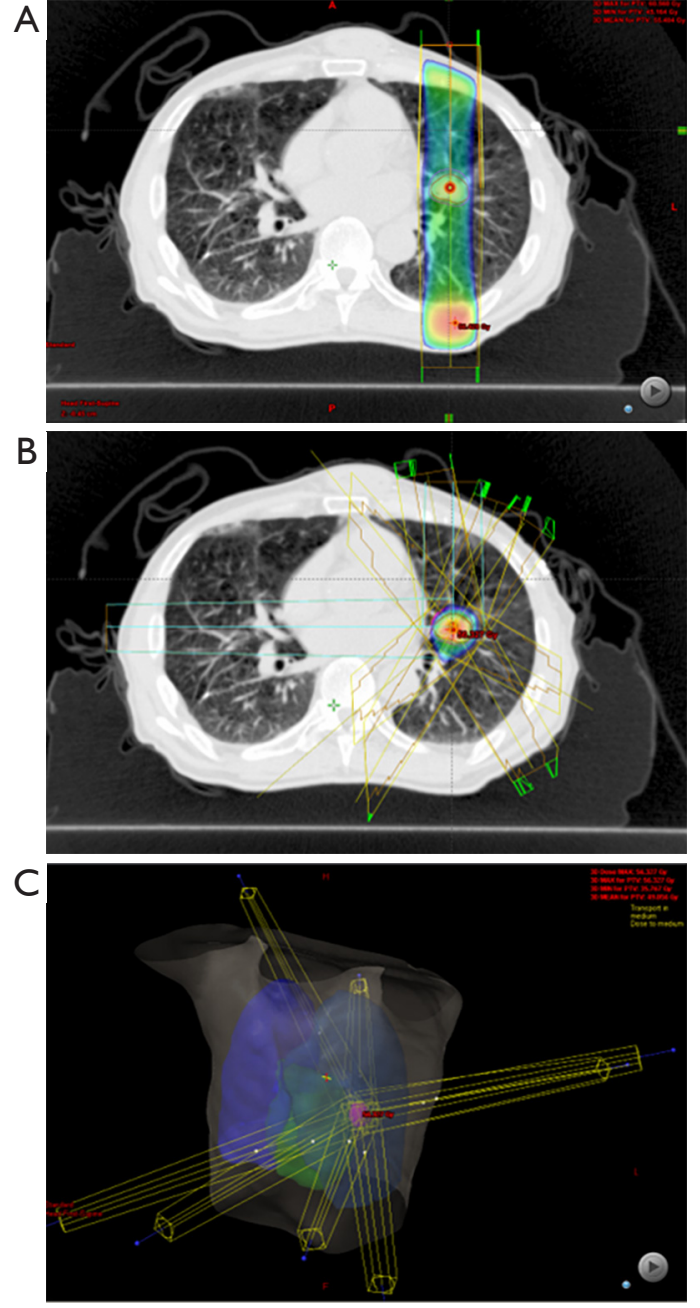

Figure 1 The differences in dose distribution between threedimensional conformal radiation (3D-CRT) (A) and stereotactic body radiation therapy (SBRT) (B) for the same left lung metastasis near the hilum of an esophageal cancer patient. (A) Using 3D-CRT shows a belt-shaped irradiation area including a part of extratumoral left lung whereas (B) SBRT can make an almost tumorshaped dose distribution which enables high dose coverage of tumor lesion and reduction of unnecessary irradiation of normal organs at the same time. (C) The overview of irradiation beams of SBRT.

surrounding the target lesions, as shown in Figure $1 \mathrm{~B}$. Therefore, radiation oncologists can deliver high radiation doses per fraction to the target lesion within a short period. A high dose per fraction is associated with a shorter treatment period and better improvement in the local tumor control than the conventionally used dose per fraction of 1.8-2 Gy (14). Stereotactic radiation was initially used for intracranial lesions, and after stereotactic irradiation for extracranial lesions using the linear accelerator was reported in 1994-1995 $(15,16)$, SBRT for NSCLC was dynamically widespread. For Stage I NSCLC, the result of SBRT was comparable to that of surgery (17).

To the best of our knowledge, there are no randomized trials for comparing SBRT with 3D-CRT in the treatment of lung metastases. However, randomized trials evaluating SBRT and 3D-CRT in early Stage NSCLC are available. In a randomized phase III trial comparing SBRT and 3D-CRT for NSCLC, no significant difference was observed, SBRT tended to have better disease control (LC) rate and less toxicity than 3D-CRT (18). Therefore, the authors concluded that SBRT should be the standard method because of its short treatment duration and reduced complications (18). Another randomized phase III trial published in 2019 showed that SBRT had significantly better LC and cancer-specific OS (19). According to these reports, SBRT/SABR may be preferred also for the treatment of lung metastases. In Japan, the Japanese insurance system covers SBRT for lung metastases that are $5 \mathrm{~cm}$ or smaller in size and with up to three lesions without extrapulmonary metastases.

An international survey on definitive SBRT for oligometastases which was published in 2017 showed that $83 \%$ of radiation oncologists began using SBRT since 2005, of which, $99 \%$ planned to continue using SBRT and $66 \%$ planned to increase the use of SBRT (20). In this survey (20), $59 \%$ of radiation oncologists who were not using SBRT for oligometastases were planning to begin SBRT soon. Therefore, to conduct SBRT for oligometastases may become one of the essential skills for radiation oncologists.

Although we did not discuss the planning of SBRT, we want to inform that several dose regimens for SBRT are available according to the site of target lesion (peripheral or central), tumor size, and the distance of normal organs from the target lesions such as chest wall, ribs, heart, esophagus, stomach, and brachial plexus. The national survey in Japan in 2009 showed that the most frequently used SBRT dose schedule for lung metastases was 48 Gy in 4 fractions followed by 50 Gy in 5 fractions and 60 Gy in 8 fractions (7). To determine the appropriate dose per fraction and accumulated total dose, previous reports showed that the biological effective dose $\alpha / \beta=10\left(\mathrm{BED}_{10}\right)$, which was calculated as "total dose" $\times(1+$ "dose per fraction"/10), should be more than 100 Gy to achieve good LC $(12,13)$. In our institution, we mainly used $42-55$ Gy in 4 fractions for $100 \%$ dose coverage $95 \%$ volume of PTV according to JCOG1408, which is undergoing clinical trial for non-operable NSCLC. 
Table 2 Treatment outcome of SBRT for oligometastatic breast cancer

\begin{tabular}{|c|c|c|c|c|c|}
\hline Author/year & Number of patients & Target & Outcomes & Toxicities & Prognostic factor \\
\hline $\begin{array}{l}\text { Scorsetti } \\
\text { et al. } \\
(23) / 2016\end{array}$ & $\begin{array}{l}33 \text { patients with less } \\
\text { than } 5 \text { metastases } \\
\text { within } 5 \mathrm{~cm} \text { in each } \\
\text { size }\end{array}$ & $\begin{array}{l}\text { Total of } 35 \\
\text { lesions: lung } 7 \text {; } \\
\text { liver } 28\end{array}$ & $\begin{array}{l}\text { Median PFS } 11 \text { months; } 1 \text {-year LC } \\
\text { 98\%, 2.3-year LC 90\%, CR 53.2\%; } \\
\text { PR 34\%; PD } 12.8 \% \text {; median OS } 48 \\
\text { months; 2-year OS } 66 \%\end{array}$ & $\begin{array}{l}\text { No G3-5; nausea and } \\
\text { vomiting G1-2 in 18\%; } \\
\text { G2 gastritis in one } \\
\text { patient; } \\
\text { G2 Cough in one } \\
\text { patient }\end{array}$ & $\begin{array}{l}\text { DFI >12 months; } \\
\text { hormonal receptor- } \\
\text { positive; medical } \\
\text { therapies after SBRT }\end{array}$ \\
\hline
\end{tabular}

SBRT, stereotactic body radiation therapy; PFS, progression-free survival; LC, local control; OS, overall survival; LN, lymph node; CR, complete response; PR, partial response; PD, progressive disease; G, grade.

However, sometimes we modify the dose, for example, 56 Gy in 7 fractions $\left(\mathrm{BED}_{10}=100.8\right)$ to isocenter as reference point for centrally located lesions. The appropriate dose schedule is determined by radiation oncologists according to the tumor status, patient's status, skill of the radiation therapist, and radiation equipment available at the institution. Nevertheless, nearly all dose schedules of SBRT for lung metastases are completed within 1-2 weeks.

In conclusion, SBRT may be preferred to 3D-CRT for lung oligometastases since SBRT has better LC, less complication, and shorter treatment interval that 3D-CRT, according to the randomized phase III trials. However, these trials studied early-stage NSCLC and not lung oligometastases.

\section{Treatment outcomes and toxicities of SBRT/ SABR for lung oligometastases}

Several studies reported the usefulness of SBRT for oligometastases from primary organs, however, only a few articles focused on oligometastatic breast cancer and these articles were summarized in Table 2. One study reported that SBRT for lung/liver oligometastases from breast cancer showed good LC rate: 1-year LC 98\%, 2-3 years of LC $90 \%$, complete response $53.2 \%$, partial response $34 \%$, and progressive disease $12.8 \%$. Despite good LC, median PFS (11 months) and median OS (48 months) were not satisfactory (23). No grade 3-4 toxicities according to common terminology criteria for adverse events (CTCAE) were observed (23). Moreover, they showed that disease-free interval (DFI) between the first diagnosis of breast cancer and appearance of metastases was longer than 12 months, and hormone-positive and systemic chemotherapy after SBRT was related to good prognosis (23). Milano et al. (21) evaluated the treatment outcomes for lung/liver/bone/ lymph nodes (LN) oligometastases from breast cancer and reported 2-year PFS 44\%, 4-year PFS 38\%, 2-year LC $89 \%$, and 4-year OS 59\%. Moreover, one metastatic lesion, small tumor volume, metastases limited to bone, and stable or regressing lesions prior to SBRT were correlated with a good prognosis. However, other reports in which 
primary lesion was not limited breast cancer and treatment outcomes were compared between breast cancer and nonbreast cancer showed that oligometastatic breast cancer had a tendency for a good prognosis, but it was not significantly different than non-breast cancer $(8,24,25)$.

Although only a few reports on oligometastatic breast cancer are available, a large number of reports on SBRT for lung metastases from any primary organs are available. Several reports $(9,13,24,26-32)$ showed a high LC rate and low toxicity of SBRT/SABR for lung oligometastases from any primary cancer. The 1- and 2-year LC rate was $89.1-$ $100 \%$ and $77.9-96 \%$, respectively, and higher than Grade 2 pneumonitis was observed in $2.6-15 \%$. In these reports, favorable PFS and OS were reported, although they were strongly influenced by primary site, chemotherapy before and after SBRT, and the definition of "oligometastases" in each institution. Hence, evaluating and interpreting SBRT benefits for survival is difficult to compare between these reports. However, the SABR-COMET phase II trial compared SBRT with standard-of-care palliative treatment and showed that SBRT improved the OS and PFS (33). Additionally, SABR improved patient-reported QOL (25). Hence, SBRT for lung oligometastases may improve LC, survival benefit, and QOLs. According to previous studies, long DFI $(13,30,32,34)$, primary tumor histology $(8,13,24,25,30)$, pretreatment performance status $(8,13)$, maximum diameter $(8,13)$, pre-SBRT chemo $(8)$, and number of metastases $(8,13)$ were associated with good prognosis.

As previously described, severe toxicities related to SBRT for peripheral lesions were reported to be rare. However, we sometimes experience severe toxicity, and especially radiation pneumonitis is a potentially fatal adverse event. Approximately $10 \%$ of SBRT patients develop have symptomatic pneumonitis which is greater than Grade 2 in CTCAE (35-37), Grade 3 pneumonitis and Grade 5 fatal respiratory toxicities were observed in $2-5 \%(36,38)$ and $0.02-0.9 \%$ patients $(7,29,39)$, respectively. It is difficult to predict accurately which patients will develop severe lung toxicity. However, several factors were related to lung toxicity such as the big size of metastatic lesions $(37,39,40)$, being female (37), smoking (37), being elderly (40), and high lung dose-volume exposure $(37,40)$. Lesions bigger than $2-3 \mathrm{~cm}$ was associated with high risk $(39,40)$. Additionally, previous reports of $3 \mathrm{D}$-CRT for thoracic showed poor nutrition (41) and several kinds of concurrent chemotherapy such as concomitant carboplatin/paclitaxel (42), gemcitabine (43), and irinotecan (43) were associated with increased risk of radiation pneumonitis. In addition to radiation pneumonitis, stenosis or perforation of trachea/ bronchus, rib fracture, perforation of the esophagus or gastric, bronchial plexopathy, pleural/ pericardial effusion, radiation dermatitis and spinal code injury are complications that may be related to SBRT.

Additionally, the location of lung metastases is important to avoid severe toxicity. If the tumor is centrally located, that is, within a $2 \mathrm{~cm}$ zone around the proximal bronchial tree, or if the PTV attached to the mediastinal or pericardial pleura (44), the risk of treatment-related severe adverse events such as pneumonitis, bronchial stenosis, pericardial effusion, dyspnea, and hemoptysis which sometimes cause death, is increased, and these complications may occur several months or years after SBRT $(10,11)$. The previous reports showed that $20-33 \%$ of patients with central lesion will develop Grade 3-5 adverse events $(10,11)$. However, though the risk of complication is higher in central lesions than in peripheral lesions, the tumor control rate is not different between central and peripheral lesions. The 2-year LC was $85.3-100 \%$ in both cases $(11,45,46)$. However, several reports showed that SBRT can be administered for central lesions with a reduced complication rate (46-48). Although there is not sufficient evidence for the safe administration of SBRT in central lesions, centrally located metastases have the potential to affect the QOL such as severe cough, shortness of breaths, and in some cases, hemoptysis according to tumor progression. Therefore, EBRT in this area should be further investigated.

Radiation pneumonitis does not usually occur during the irradiation period. One report showed that the median time to diagnose radiation pneumonitis was 5 months (range: 1.5-9 months) (35). Therefore, continuous follow-up for symptoms is essential. If patients who were treated by SBRT complain of dry cough, shortness of breath, low-grade fever, or show a pulmonary infiltrate on chest X-ray, physicians should take radiation pneumonitis into consideration and examine further as needed. In addition to imaging modalities such as X-ray and computed tomography (CT), serum KL-6 is a useful predictive marker for the early detection of radiation pneumonitis $(49,50)$.

\section{Follow-up procedure after radiation therapy}

Since SBRT causes lung changes such as radiation pneumonitis and radiation fibrosis scar of treated lesions, it is sometimes difficult to differentiate local recurrence from lung change. However, it is important to determine local recurrence, especially in patients who can be treated with 
Table 3 Summarization of previous reports on radiographic change observed on CT after SBRT

\begin{tabular}{|c|c|c|c|c|c|c|}
\hline $\begin{array}{l}\text { Months after } \\
\text { SBRT }\end{array}$ & \multicolumn{3}{|c|}{ Early changes mainly caused by pneumonitis } & \multicolumn{3}{|c|}{ Chronic changes mainly caused by fibrosis } \\
\hline $\begin{array}{l}\text { Radiographic } \\
\text { change on CT }\end{array}$ & $\begin{array}{l}\text { Almost no } \\
\text { patient has a } \\
\text { pulmonary } \\
\text { reaction (35) }\end{array}$ & $\begin{array}{l}\text { Tumor decrease in } \\
\text { size is sometimes } \\
\text { observed, and new } \\
\text { GGO or spotted- } \\
\text { streaky condensation } \\
\text { near targets is } \\
\text { observed (35) }\end{array}$ & $\begin{array}{l}\text { Radiation } \\
\text { pneumonitis } \\
\text { without symptoms } \\
\text { occur most } \\
\text { frequently (38) }\end{array}$ & & $\begin{array}{l}\text { Consolidation moves } \\
\text { toward hilum or } \\
\text { pleura because of } \\
\text { shrinking of the } \\
\text { opacity and gradually } \\
\text { shrank (35) }\end{array}$ & $\begin{array}{l}\text { Fixed consolidation } \\
\text { as solid or lined with } \\
\text { opacities; fibrotic } \\
\text { remodeling process } \\
\text { continues for years }\end{array}$ \\
\hline $\begin{array}{l}\text { Characteristics } \\
\text { of local } \\
\text { recurrence }\end{array}$ & \multicolumn{3}{|c|}{$\begin{array}{l}\text { Because new lung changes sometimes occurred related } \\
\text { to SBRT in the early phase after treatment, it is difficult to } \\
\text { differentiate between local recurrence and a pulmonary } \\
\text { injury }\end{array}$} & $\begin{array}{l}\text { The timing of } \\
\text { almost local } \\
\text { recurrence is } \\
\text { identified }(35,38)\end{array}$ & \multicolumn{2}{|c|}{$\begin{array}{l}\text { Increasing size in consolidation after } \\
12 \text { months was highly suspected of local } \\
\text { recurrence (52) }\end{array}$} \\
\hline
\end{tabular}

$\mathrm{CT}$, computed tomography; SBRT, stereotactic body radiation therapy; ${ }^{18} \mathrm{FDG}$-PET, ${ }^{18} \mathrm{~F}$-fluorodeoxyglucose positron emission tomography.

further local therapy like re-irradiation, surgical resection, and radiofrequency ablation or systemic therapy. The success of local therapy may be interpreted differently by surgeons and radiation oncologists. The success of surgical resection defined as complete resection with an appropriate margin and no mass-like lesion observed on CT imaging after the operation. However, the success of SBRT is usually defined as "no progression of the tumors" on the followup CT, and the complete disappearance of lung metastases treated by SBRT is rare, although some degree of tumor shrinkage is often observed.

Although the rate of symptomatic radiation pneumonitis in patients treated with SBRT was low, $60-100 \%$ of patients reported some radiographic change with or without symptom (51). The shape of lung injury after SBRT is different from that after 3D-CRT, due to differences in dose distributions. The lung changes after 3D-CRT including adjuvant irradiation to the breast after conserving surgery is usually of "liner-edged shape" according to the dose contribution, and it is usually easy to recognize the shadow that occurs due to the irradiation effect. However, since the dose contribution is fitted to the target shape, the shape of lung injury after SBRT usually appears as a "mass-like shape" lesion on CT images, and it is sometimes difficult to differentiate lung change due to irradiation from local recurrence, especially in the early phase after SBRT $(38,52)$. Previous reports (52-55) showed that mass-like shape lesions were observed in $40-68 \%$ of patients after SBRT, and of these, real malignant local recurrence occurred in only 11$23 \%$. Hence, it is important to determine the natural course of lung change after SBRT using CT imaging.

The chest CT changes after SBRT reported previously are shown in Table 3. Radiographic changes are roughly divided into two types according to the time after SBRT: early radiological changes as ground-glass opacity or spotted condensation related to pneumonitis and chronic radiological changes as dense consolidation or retraction related with fibrosis (35). In the early phase after SBRT within the first 6 weeks, almost all patients have no radiographic change on CT (35), and after 1.5 months, radiographic changes become gradually distinct on CT images $(38,57)$. From 3-6 months after SBRT, ground grass opacity or spotted streaky condensation sometimes appear near the treated metastases (35), and non-symptomatic radiation pneumonitis occurs most frequently at this time (38). From 7-9 months after SBRT, early changes that appeared in 3-6 months generally disappear or change to dense consolidation (35). Subsequently, dense consolidation changes both in size and appearance due to fibrotic processes such as shrinkage, retraction, or movement of shadow toward hilum or pleura $(35,38,57)$. Usually, fibrotic changes appear within the high-dose irradiated area (35).

It is difficult to differentiate between local recurrence and pulmonary injury. However, several previous reports demonstrated the usefulness of serial CT $(52,54)$ and ${ }^{18} \mathrm{~F}$-fluorodeoxyglucose positron emission tomography 
$\left({ }^{18}\right.$ FDG-PET) $(55,56)$. On CT images, patients with continuous regression for 3 times at 3 months intervals (54) and an increase in the size of the lesion after 12 months $(52,54)$ are highly suspected with local recurrence. Malignant mass-like consolidation appears in median 7 months which is earlier than that of no malignancy in median 12 months (54). On ${ }^{18}$ FDG-PET, maximum standardized uptake value (SUVmax) could differentiate local recurrence from pulmonary injury with the sensitivity and specificity of $100 \%$ and $96-98 \%$, respectively (55). Another report showed that both SUVmax and a combination of uptake intensity of FDG with uptake shape: mass-like or non-mass-like, were useful predictors for differentiating local recurrence from radiation pneumonitis (56).

\section{Problems associated with EBRT for lung oligometastases}

As mentioned previously, SBRT for lung oligometastases was less invasive and had a comparable LC rate than surgical resection. Therefore, SBRT is widely used to treat lung oligometastases. However, it may sometimes lead to overtreatment or delay systemic therapy due to complications. Therefore, a multidisciplinary team and not an individual specialist should carefully determine whether the metastatic lesion is really "oligometastases", whether the lesion should be treated by local therapy or systemic therapy, and the risk and expected benefits of these treatments.

Another disadvantage of SBRT is that the pathological findings cannot be obtained. Patients undergoing SBRT, especially non-operable patients, are sometimes diagnosed with lung metastases on CT series, without pathological proof. However, a previous report showed that $18 \%$ of surgically resected lung or brain lesions which were considered as oligometastatic lesions of NSCLC, were benign (58). Treating such patients with SBRT would needlessly add further systemic therapy in the absence of pathological findings.

Finally, in breast cancer patients with a history of irradiation to the conserved breast, chest wall, or subclavian area as adjuvant therapy after surgical treatment for primary lesions, especially in the case of lung metastases located near the apex of the lung and front chest, the previous irradiated area should be carefully checked before administration of further SBRT.

\section{Future prospects of SBRT}

Most reports described here are retrospective studies, and the survival benefit of SBRT for lung oligometastases is not truly established. Currently, a randomized phase II/ III clinical trial for the evaluation of SBRT on PFS/OS is underway. For example, a search on Clinical Trials.gov (https://clinicaltrials.gov/) showed NCT02364557 which is a randomized phase II/III trial comparing standard-ofcare therapy with or without SBRT and/or surgical ablation for newly oligometastatic breast cancer (NRG-BR002), NCT02759783 which is a phase II/III trial of comparing SBRT and the standard care for extracranial oligometastases from breast cancer, prostate cancer, and NSCLC, and NCT02089100 which is a phase III investigation evaluating SBRT for oligometastatic breast cancer.

Radiation therapy may affect both the irradiated site and the distant site, which is sometimes referred to as the abscopal effect $(59,60)$. The most impressive case report of the abscopal effect for metastatic breast cancer was reported from Japan (61). In this report, a 64-year-old woman with breast cancer that had spread to multiple bones, lung metastases, and systemic lymph nodes metastases was treated with palliative radiation monotherapy for primary breast lesions, and some painful bone metastases without systemic therapy because of her poor performance status. However, 10 months after irradiation, her gross tumors had entirely disappeared on the ${ }^{18} \mathrm{FDG}-\mathrm{PET}$ scan, and this was strongly considered as an abscopal effect (61). Though such a remarkable abscopal effect is truly rare, radiation therapy was reported to enhance immune-mediated mechanisms $(62,63)$. In stage III NSCLC, the use of adjuvant therapy with Durvalumab which is an immune checkpoint inhibitor (ICI), after concurrent chemoradiation significantly improves PFS (64), and this may be related to the enhanced immune-mediated system after radiation. Since there is no evidence for ICI monotherapy or a combination of radiation therapy and ICI in breast cancer, the immuno-effect of SBRT may have the potential of improving metastatic breast cancer treatment outcomes.

\section{Conclusions}

In conclusion, EBRT, especially SBRT/SABR, has high LC late with rare severe toxicity for lung oligometastatic breast cancer, and may potentially improve PFS and OS. Furthermore, SBRT may be able to cure oligometastatic patients. However, further investigation of oligometastatic patients is needed. In addition, the risk and expected merit of local therapy or systemic therapy should be discussed carefully by a multidisciplinary team. 


\section{Acknowledgments}

We would like to thank Editage (www.editage.com) for English language editing.

Funding: None.

\section{Footnote}

Provenance and Peer Review: This article was commissioned by the Guest Editors (Tadahiko Shien and Kaori Terata) for the series "Loco regional therapy for metastatic breast cancer" published in Translational Cancer Research. The article was sent for external peer review organized by the Guest Editors and the editorial office.

Conflicts of Interest: Both authors have completed the ICMJE uniform disclosure form (available at http://dx.doi. org/10.21037/tcr.2020.02.55). The series "Loco-regional therapy for metastatic breast cancer" was commissioned by the editorial office without any funding or sponsorship. The authors have no other conflicts of interest to declare.

Ethical Statement: The authors are accountable for all aspects of the work in ensuring that questions related to the accuracy or integrity of any part of the work are appropriately investigated and resolved.

Open Access Statement: This is an Open Access article distributed in accordance with the Creative Commons Attribution-NonCommercial-NoDerivs 4.0 International License (CC BY-NC-ND 4.0), which permits the noncommercial replication and distribution of the article with the strict proviso that no changes or edits are made and the original work is properly cited (including links to both the formal publication through the relevant DOI and the license). See: https://creativecommons.org/licenses/by-nc-nd/4.0/.

\section{References}

1. Hellman S, Weichselbaum RR. Oligometastases. J Clin Oncol 1995;13:8-10.

2. Palma DA, Olson R, Harrow S, et al. Stereotactic ablative radiotherapy versus standard of care palliative treatment in patients with oligometastatic cancers (SABR-COMET): a randomised, phase 2, open-label trial. Lancet 2019;393:2051-8.

3. Niibe $Y$, Hayakawa K. Oligometastases and oligorecurrence: The new era of cancer therapy. Jpn J Clin Oncol 2010;40:107-11.
4. Ricardi U, Badellino S, Filippi AR. Clinical applications of stereotactic radiation therapy for oligometastatic cancer patients: A disease-oriented approach. J Radiat Res 2016;57:i58-68.

5. European School of Oncology (ESO)-MBC Task Force. Metastatic breast cancer. Recommendations proposal from the European School of Oncology (ESO)-MBC Task Force. Breast 2007;16:9-10.

6. Chen H, Louie AV, Boldt RG, et al. Quality of Life After Stereotactic Ablative Radiotherapy for Early-Stage Lung Cancer: A Systematic Review. Clin Lung Cancer 2016;17:e141-9.

7. Nagata Y, Hiraoka M, Mizowaki T, et al. Survey of Stereotactic Body Radiation Therapy in Japan by the Japan 3-D Conformal External Beam Radiotherapy Group. Int J Radiat Oncol Biol Phys 2009;75:343-7.

8. Fode MM, Høyer M. Survival and prognostic factors in 321 patients treated with stereotactic body radiotherapy for oligo-metastases. Radiother Oncol 2015;114:155-60.

9. Rusthoven KE, Kavanagh BD, Burri SH, et al. MultiInstitutional Phase I/II Trial of Stereotactic Body Radiation Therapy for Lung Metastases. J Clin Oncol 2009;27:1579-84.

10. Timmerman R, McGarry R, Yiannoutsos C, et al. Excessive toxicity when treating central tumors in a phase II study of stereotactic body radiation therapy for medically inoperable early-stage lung cancer. J Clin Oncol 2006;24:4833-9.

11. Song SY, Choi W, Shin SS, et al. Fractionated stereotactic body radiation therapy for medically inoperable stage I lung cancer adjacent to central large bronchus. Lung Cancer 2009;66:89-93.

12. Onishi $H$, Shirato H, Nagata $Y$, et al. Hypofractionated stereotactic radiotherapy (HypoFXSRT) for stage I non-small cell lung cancer: Updated results of 257 patients in a Japanese multi-institutional study. J Thorac Oncol 2007;2:S94-100.

13. Rieber J, Streblow J, Uhlmann L, et al. Stereotactic body radiotherapy (SBRT) for medically inoperable lung metastases-A pooled analysis of the German working group "stereotactic radiotherapy." Lung Cancer 2016;97:51-8.

14. Mehta M, Scrimger R, Mackie R, et al. A new approach to dose escalation in non-small-cell lung cancer. Int J Radiat Oncol Biol Phys 2001;49:23-33.

15. Blomgren H, Lax I, Näslund I, et al. Stereotactic high dose fraction radiation therapy of extracranial tumors using an accelerator: Clinical experience of the first thirty-one patients. Acta Oncol 1995;34:861-70.

16. Uematsu M, Fukui T, Shioda A, et al. A dual computed 
tomography linear accelerator unit for stereotactic radiation therapy: a new approach without cranially fixated stereotactic frames. Int J Radiat Oncol Biol Phys 1996;35:587-92.

17. Onishi H, Shirato H, Nagata Y, et al. Stereotactic body radiotherapy (SBRT) for operable Stage i non-small-cell lung cancer: Can SBRT be comparable to surgery? Int J Radiat Oncol Biol Phys 2011;81:1352-8.

18. Nyman J, Hallqvist A, Lund JÅ, et al. SPACE - A randomized study of SBRT vs conventional fractionated radiotherapy in medically inoperable stage I NSCLC. Radiother Oncol 2016;121:1-8.

19. Ball D, Mai GT, Vinod S, et al. Stereotactic ablative radiotherapy versus standard radiotherapy in stage 1 nonsmall-cell lung cancer (TROG 09.02 CHISEL): a phase 3 , open-label, randomised controlled trial. Lancet Oncol 2019;20:494-503.

20. Lewis SL, Porceddu S, Nakamura N, et al. Definitive Stereotactic Body Radiotherapy (SBRT) for Extracranial Oligometastases. Am J Clin Oncol 2017;40:418-22.

21. Milano MT, Zhang H, Metcalfe SK, et al.

Oligometastatic breast cancer treated with curative-intent stereotactic body radiation therapy. Breast Cancer Res Treat. 2009;115:601-8.

22. Milano MT, Katz AW, Zhang H, et al. Oligometastases treated with stereotactic body radiotherapy: Long-term follow-up of prospective study. Int J Radiat Oncol Biol Phys 2012;83:878-86.

23. Scorsetti M, Franceschini D, De Rose F, et al. Stereotactic body radiation therapy: A promising chance for oligometastatic breast cancer. Breast 2016;26:11-7.

24. Osti MF, Carnevale A, Valeriani M, et al. Clinical outcomes of single dose stereotactic radiotherapy for lung metastases. Clin Lung Cancer 2013;14:699-703.

25. Sutera P, Clump DA, Kalash R, et al. Initial Results of a Multicenter Phase 2 Trial of Stereotactic Ablative Radiation Therapy for Oligometastatic Cancer. Int J Radiat Oncol Biol Phys 2019;103:116-22.

26. Okunieff P, Petersen AL, Philip A, et al. Stereotactic Body Radiation Therapy (SBRT) for lung metastases. Acta Oncol 2006;45:808-17.

27. Siva S, MacManus M, Ball D. Stereotactic radiotherapy for pulmonary oligometastases: A systematic review. J Thorac Oncol 2010;5:1091-9.

28. Norihisa Y, Nagata Y, Takayama K, et al. Stereotactic Body Radiotherapy for Oligometastatic Lung Tumors. Int J Radiat Oncol Biol Phys 2008;72:398-403.

29. Oh D, Ahn YC, Seo JM, et al. Potentially curative stereotactic body radiation therapy (SBRT) for single or oligometastasis to the lung. Acta Oncol 2012;51:596-602.

30. Aoki M, Hatayama Y, Kawaguchi H, et al. Stereotactic body radiotherapy for lung metastases as oligo-recurrence: A single institutional study. J Radiat Res 2016;57:55-61.

31. Navarria P, Ascolese AM, Tomatis S, et al. Stereotactic body radiotherapy (sbrt) in lung oligometastatic patients: Role of local treatments. Radiat Oncol 2014;9:91.

32. Takahashi W, Yamashita H, Niibe Y, et al. Stereotactic Body Radiotherapy for Metastatic Lung Cancer as Oligo-Recurrence: An Analysis of 42 Cases. Pulm Med 2012;2012:454107.

33. Olson R, Senan S, Harrow S, et al. Quality of Life Outcomes after Stereotactic Ablative Radiotherapy (SABR) vs. Standard of Care Treatments in the Oligometastatic Setting: A Secondary Analysis of the SABR-COMET Randomized Trial. Int J Radiat Oncol Biol Phys 2019;105:943-7.

34. Inoue T, Katoh N, Onimaru R, et al. Clinical outcomes of stereotactic body radiotherapy for patients with lung tumors in the state of oligo-recurrence. Pulm Med 2012;2012:369820.

35. Guckenberger M, Heilman K, Wulf J, et al. Pulmonary injury and tumor response after stereotactic body radiotherapy (SBRT): Results of a serial follow-up CT study. Radiother Oncol 2007;85:435-42.

36. Guckenberger M, Baier K, Polat B, et al. Dose-response relationship for radiation-induced pneumonitis after pulmonary stereotactic body radiotherapy. Radiother Oncol 2010;97:65-70.

37. Baker R, Han G, Sarangkasiri S, et al. Clinical and dosimetric predictors of radiation pneumonitis in a large series of patients treated with stereotactic body radiation therapy to the lung. Int J Radiat Oncol Biol Phys 2013;85:190-5.

38. Takeda T, Takeda A, Kunieda E, et al. Radiation Injury after Hypofractionated Stereotactic Radiotherapy for Peripheral Small Lung Tumors: Serial Changes on CT. AJR Am J Roentgenol 2004;182:1123-8.

39. Inoue T, Shimizu S, Onimaru R, et al. Clinical Outcomes of Stereotactic Body Radiotherapy for Small Lung Lesions Clinically Diagnosed as Primary Lung Cancer on Radiologic Examination. Int J Radiat Oncol Biol Phys 2009;75:683-7.

40. Zhao J, Yorke ED, Li L, et al. Simple Factors Associated with Radiation-Induced Lung Toxicity after Stereotactic Body Radiation Therapy of the Thorax: A Pooled Analysis of 88 Studies Jing. Int J Radiat Oncol Biol Phys 2016;95:1357-66.

41. Hill A, Kiss N, Hodgson B, et al. Associations between nutritional status, weight loss, radiotherapy treatment 
toxicity and treatment outcomes in gastrointestinal cancer patients. Clin Nutr 2011;30:92-8.

42. Palma DA, Senan S, Tsujino K, et al. Predicting radiation pneumonitis after chemoradiation therapy for lung cancer: An international individual patient data meta-analysis. Int J Radiat Oncol Biol Phys 2013;85:444-50.

43. Mehta V. Radiation pneumonitis and pulmonary fibrosis in non-small-cell lung cancer: Pulmonary function, prediction, and prevention. Int J Radiat Oncol Biol Phys 2005;63:5-24.

44. Kong FM, Ritter T, Quint DJ, et al. Consideration of dose limits for organs at risk of thoracic radiotherapy: Atlas for lung, proximal bronchial tree, esophagus, spinal cord, ribs, and brachial plexus. Int J Radiat Oncol Biol Phys 2011;81:1442-57.

45. Joyner M, Salter BJ, Papanikolaou N, et al. Stereotactic body radiation therapy for centrally located lung lesions. Acta Oncol 2006;45:802-7.

46. Chaudhuri AA, Tang C, Binkley MS, et al. Stereotactic ablative radiotherapy (SABR) for treatment of central and ultra-central lung tumors. Lung Cancer 2015;89:50-6.

47. Rowe BP, Boffa DJ, Wilson LD, et al. Stereotactic body radiotherapy for central lung tumors. J Thorac Oncol 2012;7:1394-9.

48. Modh A, Rimner A, Williams E, et al. Local control and toxicity in a large cohort of central lung tumors treated with stereotactic body radiation therapy. Int J Radiat Oncol Biol Phys 2014;90:1168-76.

49. Goto K, Kodama T, Sekine I, et al. Serum levels of KL-6 are useful biomarkers for severe radiation pneumonitis. Lung Cancer 2001;34:141-8.

50. Hara R, Itami J, Komiyama T, et al. Serum Levels of KL-6 for Predicting the Occurrence of Radiation Pneumonitis after Stereotactic Radiotherapy for Lung Tumors. Chest 2004;125:340-4.

51. Bradley J. Radiographic response and clinical toxicity following SBRT for stage I lung cancer. J Thorac Oncol 2007;2:S118-24.

52. Matsuo Y, Nagata Y, Mizowaki T, et al. Evaluation of mass-like consolidation after stereotactic body radiation therapy for lung tumors. Int J Clin Oncol 2007;12:356-62.

53. Takeda A, Kunieda E, Takeda T, et al. Possible misinterpretation of demarcated solid patterns of radiation fibrosis on ct scans as tumor recurrence in patients receiving hypofractionated stereotactic radiotherapy for lung cancer. Int J Radiat Oncol Biol Phys 2008;70:1057-65.

54. Dunlap NE, Yang W, McIntosh A, et al. Computed tomography-based anatomic assessment overestimates local tumor recurrence in patients with mass-like consolidation after stereotactic body radiotherapy for early-stage non-small cell lung cancer. Int J Radiat Oncol Biol Phys 2012;84:1071-7.

55. Takeda A, Kunieda E, Fujii H, et al. Evaluation for local failure by $18 \mathrm{~F}-\mathrm{FDG}$ PET/CT in comparison with CT findings after stereotactic body radiotherapy (SBRT) for localized non-small-cell lung cancer. Lung Cancer 2013;79:248-53.

56. Nakajima N, Sugawara Y, Kataoka M, et al. Differentiation of tumor recurrence from radiation-induced pulmonary fibrosis after stereotactic ablative radiotherapy for lung cancer: Characterization of 18F-FDG PET/CT findings. Ann Nucl Med 2013;27:261-70.

57. Aoki T, Nagata Y, Negoro Y, et al. Evaluation of Lung Injury after Three-dimensional Conformal Stereotactic Radiation Therapy for Solitary Lung Tumors: CT Appearance. Radiology 2004;230:101-8.

58. Endo C, Hasumi T, Matsumura Y, et al. A prospective study of surgical procedures for patients with oligometastatic non-small cell lung cancer. Ann Thorac Surg 2014;98:258-64.

59. Wattenberg MM, Fahim A, Ahmed MM, et al. Unlocking the Combination: Potentiation of Radiation-Induced Antitumor Responses with Immunotherapy. Radiat Res 2014;182:126-38.

60. Mole RH. Whole body irradiation; radiobiology or medicine? Br J Radiol 1953;26:234-41.

61. Azami A, Suzuki N, Azami Y, et al. Abscopal effect following radiation monotherapy in breast cancer: A case report. Mol Clin Oncol 2018;9:283-6.

62. Lee Y, Auh SL, Wang Y, et al. Therapeutic effects of ablative radiation on local tumor require $\mathrm{CD} 8+\mathrm{T}$ cells: Changing strategies for cancer treatment. Blood 2009;114:589-95.

63. Liang H, Deng L, Chmura S, et al. RadiationInduced Equilibrium Is a Balance between Tumor Cell Proliferation and T Cell-Mediated Killing. J Immunol 2013;190:5874-81.

64. Antonia SJ, Villegas A, Daniel D, et al. Durvalumab after chemoradiotherapy in stage III non-small-cell lung cancer. N Engl J Med 2017;377:1919-29.

Cite this article as: Wada Y, Hashimoto M. Modern evidence and future prospects of external body radiation therapy for lung oligometastases of breast cancer. Transl Cancer Res 2020;9(8):50775086. doi: $10.21037 /$ tcr.2020.02.55 